\title{
Aberrant Expression of CD Markers in Cases with Acute Leukemia in Sohag University Hospital
}

\author{
Noura F. Abdullah, Hasnaa A. Aboelwafa, Ahmed A. Allam and Ahmed M. \\ Mohamed \\ Department of Clinical Pathology, Faculty of Medicine, Sohag University
}

\begin{abstract}
Introduction: Acute leukemias are a group of malignancies with clinical, morphologic, immunologic, and molecular patterns and display patterns of surface antigen expression (CD antigens). Precursor cells from different blast cells in acute leukemia express different subdivisions of surface molecules many of which are now defined as cluster of differentiation (CD) antigens. Every leukemia type has specific set of CD markers, what constitute nowadays current classification of hematopoietic malignancies and the basis of diagnosis. The expression of $\mathrm{CD}$ markers on leukocytes can be determined by flowcytometry. Immunophenotyping improves both accuracy and reproducibility of acute leukemia classification. Flow cytometry is a technique used to analyze multiple phenotypic and functional parameters simultaneously within a single cell or a population of cells.

Aim of the work: Assess the prevalence of aberrant expression of CD markers in cases with acute leukemia in Sohag University Hospitals.

Patients and Methods: This study was carried out in the Clinical Pathology Department, faculty of medicine, Sohag university hospital on fifty (50) newly diagnosed acute leukemia patients who were subjected to bone marrow aspiration, their ages ranged between 1.5 to 71 years, $29(58 \%)$ of them were males and $21(42 \%)$ were females with a male to female ratio of 1.4:1, during the period from November 2015 to October 2016.

Results: B-cell acute lymphoblastic leukemia positive for the following CD markers CD10, CD19, TdT, HLA-DR and expressing CD33 and case of AML (M4-M5) expressing CD7 lymphoid marker.

Conclusion: Aberrant expression of $C D$ markers is seen in several cases of acute leukemia. The frequency of aberrant antigen expression in acute leukemia was comparable with most published international data. The presence of aberrancy helps to identify a neoplastic process.
\end{abstract}

Key words: CD Markers, Leukemia, Aberrant.

\section{Introduction}

Acute leukemias are a mixed group of malignancies with varying clinical, morphologic, immunologic, and molecular types and display characteristic forms of surface antigen expression (CD antigens). Precursor cells from different blast cells in acute leukemia express different subdivisions of surface molecules many of which are now defined as cluster of differentiation (CD) antigens. Each leukemia type has typical set of CD markers, what constitute nowadays current grouping of hematopoietic malignancies and the basis of diagnosis ${ }^{(\mathbf{1})}$.

Expression of $\mathrm{CD}$ markers on leukocytes can be determined by flow cytometry ${ }^{(1)}$.

Immunophenotyping improves the accuracy and reproducibility of acute leukemia classification. Flow cytometry is a technique used to examine multiple phenotypic and functional parameters simultaneously within a single cell or group of cells ${ }^{(2)}$.

Its ability to measure many factors on individual cells in a suspension at high speed is ideal for the study of leukemic cells. It identifies cell markers by applying monoclonal antibodies against them ${ }^{(3)}$. 
Several immunophenotypes of blast cells from cases with acute leukemia do not show the criteria of normal cellular differentiation and show unusual expression of markers of differentiation called aberrant expression of these markers. Many techniques in flow cytometric immunophenotyping improved gating strategies, and the multiparameter analytic techniques, have all dramatically improved the utility of flow cytometry in the diagnosis and grouping of leukemia ${ }^{(4)}$.

These new applications of flow cytometry immunophenotyping mainly trust on the concept that even if neoplastic cells show an excessive similarity to normal hematopoietic precursors, they frequently display aberrant phenotypes that allow their specific documentation and discrimination from normal cells, even when present at low frequencies. To a great extent, those aberrant phenotypes would be a consequence of the genetic abnormalities shown by the neoplastic cell $^{(5)}$.

\section{Aim of the work}

To assess the frequency of aberrant expression of CD markers in cases with acute leukemia in Sohag University Hospitals.

\section{Patients and Methods:}

Design: Cross sectional clinical study. Patients

This study was carried out in the Clinical Pathology Department, faculty of medicine, Sohag university hospital on fifty (50) newly diagnosed acute leukemia patients who were subjected to bone marrow aspiration, their ages ranged between 1.5 to 71 years, 29 $(58 \%)$ of them were males and 21 $(42 \%)$ were females with a male to female ratio of $1.4: 1$, during the period from November 2015 to October 2016. This study was approved by the Research and Ethical Committee at faculty of medicine, Sohag University. All subjects were informed about the aim of this study and gave written consents.

\section{Methods:}

All members of the study were subjected to the following investigations:

1. Full history taking.

2. Clinical examination.

3. Laboratory investigation:

- Complete blood count.

- BM examination.

- Flow cytometry panel for diagnosis of acute leukemia.

1- Complete blood count (CBC) using Cell-Dyn 3700 and Cell-Dyn Ruby, automated cell counters, ABBOTT diagnostic (USA), with examination of leishman-stained PB smears for differential leucocytes count and blast cells percentage.

2- BM aspiration and examination of Leishman-stained smears to detect the percentage of BM blast cells.

The FAB classification was used for the morphological classification of acute leukemia cases.

3- Immunophenotyping of blast cells in BM aspirate and peripheral blood samples using BD FACSCalibur Flow Cytometer ${ }^{(6)}$.

Diagnosis of acute leukemia was based on the presence of blast cells $\geq 20 \%$ in $\mathrm{BM}$ film according to WHO proposal, together with presence of immunophenotyping results constant with acute leukemia.

A- Sampling:

$-3 \mathrm{ml}$ of venous blood were aseptically collected from each patient, dispensed into a tube containing $\mathrm{K}$ ethylene diamine tetra-acetic acid (K-EDTA) at a concentration of 1.2 $\mathrm{mg} / \mathrm{ml}$, to be used for $\mathrm{CBC}$, preparation of Leishman-stained smears PB smears and flow cytometry if needed.

- BM aspiration was done for all patients. A part was used for spreading smears to be examined by 
Leishman while the remaining part was dispensed into sterile tube containing K-EDTA to be used for the flow cytometry .

B- Immunophenotyping of blast cells: Principle of Flow Cytometry (FMC): FCM is the measurement of numerous cell properties (cytometry) as the cells move in single file resulting in light scattering. Antibodies specific for various cellular antigens can be considered with different fluorochromes that can absorb and emit light, agreeing simultaneous multicolor flow cytometric analysis of two or more cell-associated antigens.

Reagents:

- Sheath fluid.

- Phosphate buffered saline (PBS) $(8.0 \mathrm{~g} / \mathrm{L} \mathrm{NaCl}, 0.2 \mathrm{~g} / \mathrm{L} \mathrm{kcl}, 1.15 \mathrm{~g} / \mathrm{L}$ $\mathrm{NaH} 2 \mathrm{PO} 4$ and $0.2 \mathrm{~g}$ kH2PO4) added to $100 \mathrm{~mL}$ of distilled water with $\mathrm{pH}$ adjusted at $7.3 \pm 0.2$.

- Lysing solution (1.5 $\mathrm{mmol} / \mathrm{L}$ $\mathrm{NH} 4 \mathrm{Cl}, 100 \mathrm{mmol} / \mathrm{L} \mathrm{KHCO} 3$ and $10 \mathrm{mmol} / \mathrm{L}$ tetra Na-EDTA) made up to 1 liter with distilled water, $\mathrm{pH}$ adjusted at 7.2.

- Negative isotypic control (appropriately labelled according to the MoAbs used) for determining the non-specific binding of MoAbs.

- MoAbs supplied by BD

Bioscience, United states.

Procedure:

$\mathrm{BM}$ or $\mathrm{PB}$ samples were processed within 6 hours of collection.

A minimum of 5000 events were studied. Gating was done on the blast cell population based on forward and side scatter properties.

\section{Data interpretation:}

The percentage of blast cells positive for the relevant studied marker was determined as a percentage from the gated blast cells population. The negative isotypic control was set at $2 \%$. Cells were considered positive for a certain marker when $\geq 20 \%$ of cells expressed.

\section{Inclusion criteria:}

Cases with newly diagnosed acute leukemias.

\section{Exclusion criteria:}

Cases with Chronic leukemias, Lymphoma or Leukemic phase of lymphoma will be excluded from the study.

\section{Results}

The mean age in group I was $36.23 \pm 22.84$ years and ranged from 3.5 to 82 , in group II it was $18.33 \pm 22.22$ and ranged form 1.5 to 62 , and group III it was $17.33 \pm 6.04$ and ranged from 11 to 23 years. Sex distribution in group (I): showed that 19 patients $(59.38 \%)$ were males, and 13 patients $(40.63 \%)$ were females, in group (II) 8 patients $(53.33 \%)$ were males, and 7 patients $(46.67 \%)$ were females and group (III) showed that 2 patients $(66.67 \%)$ were males and 1 patient $(33.33 \%)$ was a female.

By examination: In group I, 14 patients $(43.75 \%)$ had no splenomegaly, 13 patients $(40.63 \%)$ had mild splenomegaly, 3 patients $(9.38 \%)$ had moderate splenomegaly and 2 patients $(6.25 \%)$ had marked splenomegaly, in group (II) 5 patients $(33.33 \%)$ had no splenomegaly, 9 patients $(60.00 \%)$ had mild splenomegaly and 1 patient $(6.67 \%)$ had moderate splenmegaly, and in group (III) 2 patients (66.67\%) had no splenomegaly and 1 patient $(33.33 \%)$ had mild splenomegaly, by comparing splenic affection between three studied groups revealed no stastical significance.

Regarding hepatomegaly, in group I 20 patients $(62.50 \%)$ had no hepatomegaly while 12 patients $(37.50 \%$ ) had mild hepatomegaly, in group (II) 10 patients $(66.67 \%)$ had no hepatomegaly while 5 patients $(33.33 \%)$ had mild hepatomegaly, and in group (III) $100 \%$ of patients had no hepatomegaly, by comparing liver affection between three studied groups revealed no stastical significance. 
Lymph node examination: 17 patients $(53.13 \%)$ in group I had no lymph node affection while 15 patients $(46.88 \%$ ) had mild lymphadenopathy, in group (II) no lymph node affection was observed in 8 patients (53.33\%) while 7 patients (46.67\%) were presented with mild lymphadenopathy, and in group (III) no lymph node affection was observed in 2 patients $(66.67 \%)$ while 1 patient $(33.33 \%)$ was presented with mild lymphadenopathy, by comparing lymph node affection between three studied groups revealed no statistical significance,

Laboratory investigations: In group (I): Haemoglobin level ranged from $2.9 \mathrm{~g} / \mathrm{dl}$ to $10.6 \mathrm{~g} / \mathrm{dl}$ with mean $\pm \mathrm{SD}$ value6.13 \pm 1.74 , platelet count ranged from $3 \times 109 / \mathrm{L}$ to $109 \times 109 / \mathrm{L}$ with mean $\pm \mathrm{SD}$ value $41.78 \pm 26.84$, WBCs count ranged from $0.8 \times 109 / \mathrm{L}$ to $290 \times 109 / \mathrm{L}$ with mean \pm SD value $47.79 \pm 66.38$.

In group (II): Haemoglobin level ranged from $3.8 \mathrm{~g} / \mathrm{dl}$ to $11.7 \mathrm{~g} / \mathrm{dl}$ with mean $\pm \mathrm{SD}$ value $6.57 \pm 2.11$, platelet count ranged from $8 \times 109 / \mathrm{L}$ to $82 \times 109 / \mathrm{L}$ with mean $\pm \mathrm{SD}$ value $40.87 \pm 24.29$, WBCs count ranged from $2.8 \times 109 / \mathrm{L}$ to $226 \times 109 / \mathrm{L}$ with mean \pm SD value $51.23 \pm 63.37$.

In group (III): Haemoglobin level ranged from $7.3 \mathrm{~g} / \mathrm{dl}$ to $13.3 \mathrm{~g} / \mathrm{dl}$ with mean $\pm \mathrm{SD}$ value $10.1 \pm 3.01$, platelet count ranged from $24 \times 109 / \mathrm{L}$ to $151 \times 109 / \mathrm{L}$ with mean $\pm \mathrm{SD}$ value $77 \pm 66.05$, WBCs count ranged from $54 \times 109 / \mathrm{L}$ to $144 \times 109 / \mathrm{L}$ with mean \pm SD value $92.00 \pm 46.60$.

Regarding Blast cells: In group (I): Blast cells (PB) ranged from 12\% to 90\% with mean \pm SD value $58.03 \pm 23.40$. In group (II): Blast cells (PB) ranged from $22 \%$ to $87 \%$ with mean \pm SD value $64.67 \pm 18.55$ and in group (III): Blast cells (PB) ranged from $65 \%$ to $73 \%$ with mean \pm SD value $69.33 \pm 4.04$.

\section{Blast cells in bone marrow (BM):}

Group (I): Blast cells (BM) ranged from $50 \%$ to $90 \%$ with mean \pm SD value 75.31 \pm 9.69 . Group (II): Blast cells (BM) ranged from $65 \%$ to $91 \%$ with mean $\pm \mathrm{SD}$ value $78 \pm 9.66$. Group (III): Blast cells (BM) ranged from $87 \%$ to $97 \%$ with mean \pm $\mathrm{SD}$ value $92.33 \pm 5.03$. Comparing group (I) with group (II) revealed no statistical significance with $(\mathrm{P}$ value $=1.00)$. Comparing group (I) with group (III) revealed statistical significance with $(\mathrm{P}$ value $=0.02$ ). Comparing group (II) with group (III) revealed no statistical significance with $(\mathrm{P}$ value $=0.07)$.

Distribution of aberrant CD markers in studied populations:

Shown in Table (1).

Table (1): Distribution of aberrant CD markers in studied populations:

\begin{tabular}{|c|c|c|c|}
\hline Variables & $\begin{array}{c}\text { AML } \\
\mathbf{N}=32\end{array}$ & $\begin{array}{c}\text { B-ALL } \\
\mathbf{N}=\mathbf{1 5}\end{array}$ & $\begin{array}{c}\text { T-ALL } \\
\mathbf{N}=3\end{array}$ \\
\hline HLADR & $17(53.13 \%)$ & - & $1(33.33 \%)$ \\
\hline CD19 & $3(9.38 \%)$ & - & $1(33.33 \%)$ \\
\hline CD13 & - & $3(20.00 \%)$ & $1(33.33 \%)$ \\
\hline CD33 & - & $2(13.33 \%)$ & $1(33.33 \%)$ \\
\hline CD14 & - & $1(6.67 \%)$ & - \\
\hline CD5 & $2(6.25 \%)$ & - & - \\
\hline CD7 & $9(28.13 \%)$ & - & - \\
\hline CD117 & - & $1(6.67 \%)$ & - \\
\hline CD3 & $1(3.13 \%)$ & - & - \\
\hline TdT & $2(6.25 \%)$ & - & - \\
\hline
\end{tabular}


Table (2): Frequency of lymphoid marker in subclass of AML patient in FAB study

\begin{tabular}{|c|c|c|c|c|c|c|c|c|}
\hline \multirow{2}{*}{ FAB } & \multicolumn{4}{|c|}{ T cell markers } & \multicolumn{4}{c|}{ B cell markers } \\
\cline { 2 - 10 } & CD5 & CD2 & CD3 & CD7 & CD19 & CD20 & CD22 & CD10 \\
\hline M0 & 0 & 0 & 0 & 0 & 0 & 0 & 0 & 0 \\
\hline M1 & 0 & 0 & 0 & 0 & 0 & 0 & 0 & 0 \\
\hline M2 & 0 & 0 & 1 & 1 & 0 & 0 & 0 & 0 \\
\hline M3 & 0 & 0 & 0 & 2 & 0 & 0 & 0 & 0 \\
\hline M4- & 2 & 0 & 0 & 3 & 3 & 0 & 0 & 0 \\
M5 & & & & & & & & \\
\hline M6 & 0 & 0 & 0 & 2 & 0 & 0 & 0 & 0 \\
\hline M7 & 0 & 0 & 0 & 1 & 0 & 0 & 0 & 0 \\
\hline
\end{tabular}

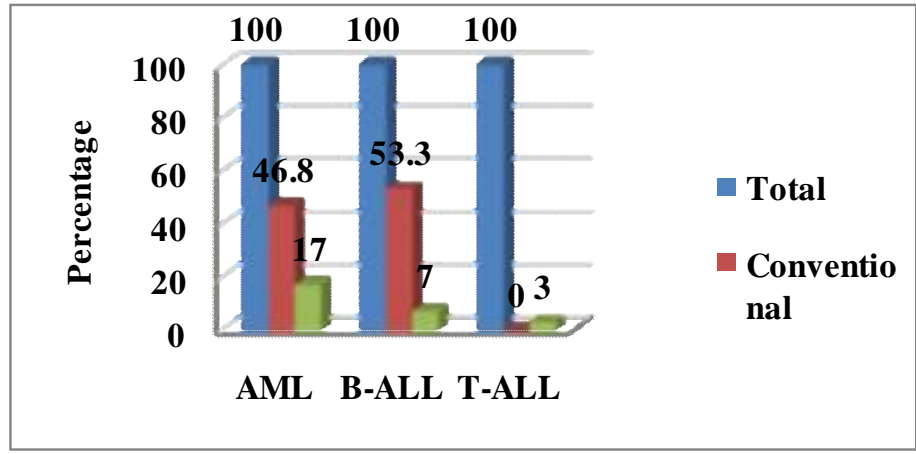

Figure (1): Frequency of aberrant and conventional antigens expression in acute leukemia $(\mathrm{AML}=$ Acute myeloid leukemia, B-ALL= B-cell acute lymphoblastic leukemia, T-ALL= T-cell acute lymphoblastic leukemia).

\section{Discussion}

In the present study, the immunophenotypes of blasts from 50 patients with acute leukemia were studied to determine the frequency of aberrant markers in this group of patients. The patients were classified into three groups: group I: Acute myeloid leukemia patients (AML) consisted of 32 patients), group II: BAcute lymphoblastic leukemia patients (B-ALL) consisted of 15 patients and group III: T-Acute lymphoblastic leukemia patients (T-ALL) consisted of 3 patients. The age range of all cases at the time of diagnosis was 1.5 to 82 years with mean age of 41 years, with a wide SD of more than 22 years, around $31 \%$ were Adults (>20 years), $22 \%$ were young adults (12-20 years) and $47 \%$ were children ( $<12$ years $)$. This was in consistence with results of study of Haferlach et al. ${ }^{(7)}$ as the age of patients at the time of diagnosis ranged from 1 year to 78 years.

Group (I) ages ranged from 3.5 to 82 years with mean of 36.23 years with SD value of 22.84 years. Group (II) ages ranged from 1.5 to 62 years with mean of 18.33 years with SD value of 22.22 years. Group (III) ages ranged from 11 to 23 years with mean of 17 years with SD value of 6 years.

The sex distribution in group (I) showed that 19 patients $(59.38 \%)$ were males, and 13 patients $(40.63 \%)$ were females, in group (II) showed that 8 patients $(53.33 \%)$ were males, and 7 patients $(46.67 \%)$ were females and in group (III) showed that 2 patients $(66.67 \%)$ were males and 1 patient $(33.33 \%)$ was a female. Male to female ratio equals $1.4: 1$, which is similar to 
that reported by other international reports ${ }^{(8)}$. Out of 50 cases, $32(64 \%)$ cases were of AML and $15(30 \%)$ of BALL and $3(6 \%)$ of T-ALL.

By examination of group I: 14 patients $(43.75 \%)$ had no splenomegaly, 13 patients $(40.63 \%)$ had mild splenomegaly, 3 patients $(9.38 \%)$ had moderate splenomegaly and 2 patients $(6.25 \%)$ had marked splenomegaly, of group II: 5 patients (33.33\%) with had no splenomegaly, 9 patients $(60 \%)$ had mild splenomegaly and 1 patient $(6.67 \%)$ had moderate splenmegaly and of group III: 2 patients $(66.67 \%)$ had no splenomegaly and 1 patient $(33.33 \%)$ had mild splenomegaly. Comparing splenic affection between three studied groups revealed no stastical significance.

Regarding hepatomegaly, group I: 20 patients $(62.50 \%)$ had no hepatomegaly while 12 patients $(37.50 \%)$ had mild hepatomegaly, of group II: 10 patients $(66.67 \%)$ had no hepatomegaly while 5 patients $(33.33 \%)$ had mild hepatomegaly, as for group III: $100 \%$ of patients had no hepatomegaly. Comparing liver affection between three studied groups revealed no stastical significance. Our results in consistant with Hoelzer et al. ${ }^{(9)}$ who reported that both ALL and CLL can be present with hepatic involvement.

As regarding examination for lymphadenopathy, of group I: 17 patients $(53.13 \%)$ had no lymph node affection while 15 patients $(46.88 \%)$ had mild lymphadenopathy, of group II: no lymph node affection was observed in 8 patients $(53.33 \%)$ while 7 patients $(46.67 \%)$ were presented with mild lymphadenopathy, and of group III: no lymph node affection was observed in 2 patients $(66.67 \%)$ while 1 patient $(33.33 \%)$ was presented with mild lymphadenopathy. Comparing lymph node affection between three studied groups revealed no statistical significance.
As for CBC, comparing WBCs count in the studied groups revealed no statistical significance. Regarding haemoglobin level, comparing group (I) with group (II) revealed no statistical significance while comparing group (I) with group (III), group (II) with group (III) both were of statistical significance. Comparing PLT count in three studied groups revealed no statistical significance in contrast with the results found by Weinberg et al. ${ }^{(10)}$. Regarding peripheral blood blast cell count, comparing the studied groups revealed no statistical significance. As for bone marrow blast cell count only comparison of group (I) with group (III) revealed statistical significance.

In this research, FAB studies on AML patient showed that AML-M4-M5 was the most common subtype of AML, which is in contrast with Lowenberg et al. ${ }^{(11)}$ findings who reported that AMLM2 was the most common. The incidence of abnormal phenotypes has been reported in literature for both AML and ALL with variable frequency ranging from $11 \%$ to $88 \%{ }^{(\mathbf{1 2})}$. Our study reports the incidence of aberrant phenotypes in all the studied cases of acute leukemia to be about 54\% compared to $30 \%$ as reported by Khurram et al $2010^{(22)}$, So more than $40 \%$ of the studied cases exhibited conventional B-cell, T-cell and myeloid immunophenotypes.

Differences in incidence of aberrant phenotypes may be attributed to numerous factors including use of a large difference of monoclonal antibody (MoAbs) panels, use of different components against the $\mathrm{CD}$ surface antigens, different cut-off levels (13), analysis of fresh or frozen cell material, change between phenotypic characteristics of blast cells of children and adults patient and geographical change in leukemia subtypes ${ }^{(8)}$.

The clinical significance of lymphoid antigen expression in AML has been 
highly controversial. Some studies have reported Ly+AML to be associated with the bad prognosis but some reported it to be associated with satisfactory prognosis whereas others suggest it to be of no prognostic value (14). Expression of lymphoid antigens in cases with AML have been found to be variable depending on the studied markers, sample size and criteria of aberrancy used (15) who included asynchronous expression of antigens also as aberrancy in AML, reported frequency as high as $88 \%$ but in their study also lymphoid antigen expression was seen in $34.2 \%$ AML. Launder et al. ${ }^{(16)}$ from Atlanta studied the incidence of one or more lymphoid antigens expression in AML which was seen in $22 \%$, This is in agreement with our results in which the aberrancy was seen in $100 \%$ of T-ALL and $46 \%$ of B-cell ALL cases and $53 \%$ of AML cases as shown in Figure(1).

Regarding the aberrant antigens expression in B- cell ALL, CD13 which is normally expressed on hematopoietic stem cells, on the mature and immature elements of the myeloid and monocytic lineages was expressed in $20 \%$ of our cases while CD33 which is a myeloid antigen was expressed in $13 \%$ of cases so that were the most common markers expressed in B-cell ALL cases.

The expression of CD33 has been reported in 3-30\% of B-ALL cases but is not related to any prognostic meaning, either in adult or in pediatric cases. In adult cases, CD33 expression is mutually exclusive with CD66c expression, and correlates with the CD10+ CD20-phenotype, the expression of CD34, and the deletion of chromosome 7 , but to neither translocation $\mathrm{t}(4 ; 11)$ nor translocation $\mathrm{t}$ $(9 ; 22){ }^{(17)}$. Our results are in accordance with that reported by Seegmiller et al. (18) who demonstrated that CD13 was the most commonly marker expressed in B-ALL followed by CD33 (Fig.3) and CD15. In contrast to Alkayed et al.
(19) at King Hussein Cancer Center in Jordan, who studied the aberrancy in Jordanian children with B-cell ALL, and found that CD33 expression being the most common followed by CD13. In T-cell ALL, CD13 and CD33 which are myeloid antigens and CD 19 which is a B-cell antigen, were the antigens expressed equally, each was $33 \%$. This is similar to that published by 2008 WHO classification which stated that CD79a may be detected in $10 \%$ of $\mathrm{T}$ ALL cases and CD13, CD33 are stated in $19-32 \%$. With regards to the paired aberrancy, our study showed that CD13 and CD33 weren't co-expressed in BALL and T-cell

Among the aberrant AML cases, we found that CD7 which is an activation and adhesion molecule, is communicated by T-cells, NK cells and stem cells, was the most common aberrant marker expressed in 9 cases $(28 \%)$ in agreement with the results of Bahia et al. ${ }^{(15)}$ and Jahedi et al. ${ }^{(\mathbf{8})}$.

CD19 was expressed in 3 (9\%) of our cases, e.g. Figure (2), compared to $28 \%$ that reported by Sarma et al. ${ }^{(12)}$ from India. Regarding the distribution of CD19 in our cases, it was expressed mainly in AML-M4 as that reported by Bahia et al. ${ }^{(15)}$.

According to TdT which is is an early lymphocyte marker included in the reorganization of $\mathrm{T}$-cell receptor genes and immunoglobulin. It is typical for lymphoblastic leukemia and lymphoma, we found 2 cases $(6 \%)$ that were positive for this nuclear marker that mainly associated with more mature AML such as M3 and M4 in contrast to that stated by Venditti et al. ${ }^{(20)}$ from Italy. CD5 is a co-stimulatory molecule found on $\mathrm{T}$ cells and $\mathrm{B}$ cells and is a main regulator of immune tolerance. In this study, CD5 was expressed in 2 cases (6\%), in M4-M5. CD3, a T-cell marker, which is involved in the signal transduction and assembly of the $\mathrm{T}$ cell receptor 
complex. In a study by Bradstock et al. (21), CD3 was positive in $7 \%$ of AML cases. While in this investigation, CD3 was positive in $3 \%$ of AML patients in M0-M2. The frequency of lymphoid markers expression in subclass of AML patient according to FAB study is summarized in table (3).

\section{Conclusion}

We conclude that aberrant expression of $\mathrm{CD}$ markers is seen in several cases of acute leukemia. The frequency of aberrant antigen expression in acute leukemia was comparable with most published international data. The presence of aberrancy helps to identify a neoplastic process.

\section{Recommendations:}

1. Aberrant expression of CD markers studying can be recommended for evaluating disease progression (prognostic factor) in acute leukemia as regard survival rate and disease relapse.

2. Aberrant expression of CD markers studying can be useful in detection of Minimal Residual Disease (MRD).

3. Further studies are recommended to confirm the correlation of aberrant phenotypes in diagnosis, prognosis and therapeutic response of acute leukemia.

4. Molecular cytogenetic studies are recommended to shed light on potential biomarkers that might play a role in pathogenesis of antigen aberrancy, prognostic stratification and possible drugable target.

\section{References}

1.Lewis RE, Cruse JM, Sanders CM, Webb RN, Suggs JL. Aberrant expression of T-cell markers in acute myeloid leukemia. Experimental and molecular pathology. 2007;83(3):462-3.

2. Strobl H, Knapp W. Myeloid cellassociated lysosomal proteins as flow cytometry markers for leukocyte lineage classification. Journal of biological regulators and homeostatic agents. 2004;18(3-4):335-9.

3. Zeleznikova $T$, Babusikova $\mathrm{O}$. The value of dot plot patterns and leukemiaassociated phenotypes in AML diagnosis by multiparameter flow cytometry. Neoplasma. 2005;52(6):51722.

4. Chang L, Zhang G, Shi H, Ye L, Jiang YM. [Clinical value of minimal residual disease detection by flow cytometry in childhood B-cell acute lymphoblastic leukemia]. Zhongguo dang dai er ke za zhi $=$ Chinese journal of contemporary pediatrics. 2014;16(12):1245-9.

5. Jani P, Qi XY, Chang H. Aberrant expression of T-cell-associated markers CD4 and CD7 on B-cell chronic lymphocytic leukemia. American journal of hematology. 2007;82(1):73-6.

6. Bennett JM, Catovsky D, Daniel MT, Flandrin G, Galton DA, Gralnick HR, et al. Proposals for the classification of the acute leukaemias. French-AmericanBritish (FAB) co-operative group. British journal of haematology. 1976;33(4):451-8.

7. Haferlach T, Kohlmann A, Schnittger S, Dugas M, Hiddemann W, Kern W, et al. Global approach to the diagnosis of leukemia using gene expression profiling. Blood. 2005;106(4):1189-98.

8. Jahedi M, Shamsasenjan K, Sanaat Z, Aliparasti M, Almasi S, Mohamadian $M$, et al. Aberrant phenotype in Iranian patients with acute myeloid leukemia. Advanced pharmaceutical bulletin. 2014;4(1):43-7.

9. Hoelzer D, Gokbuget N. New approaches to acute lymphoblastic leukemia in adults: where do we go? Seminars in oncology. 2000;27(5):54059.

10.Weinberg OK 'Seetharam M, Ren L, Seo K, Ma L, Merker JD, et al. Clinical characterization of acute myeloid leukemia with myelodysplasia-related changes as defined by the 2008 WHO classification system. Blood. 2009;113(9):1906-8.

11.Lowenberg B. Hematology in 2000 and beyond: a weather forecast. The Netherlands journal of medicine. 1999;55(6):276-9. 
12. Sarma A, Hazarika M, Das D, Kumar Rai A, Sharma JD, Bhuyan C, et al. Expression of aberrant $\mathrm{CD}$ markers in acute leukemia: a study of 100 cases with immunophenotyping by multiparameter flowcytometry. Cancer biomarkers : section A of Disease markers. 2015;15(4):501-5.

13. Salem DA, Abd El-Aziz SM. Flowcytometric immunophenotypic profile of acute leukemia: mansoura experience. Indian journal of hematology \& blood transfusion : an official journal of Indian Society of Hematology and Blood Transfusion. 2012;28(2):89-96.

14. Zheng J, Wang X, Hu Y, Yang J, Liu J, $\mathrm{He} \mathrm{Y}$, et al. A correlation study of immunophenotypic, cytogenetic, and clinical features of 180 AML patients in China. Cytometry Part B, Clinical cytometry. 2008;74(1):25-9.

15. Bahia DM, Yamamoto M, Chauffaille Mde L, Kimura EY, Bordin JO, Filgueiras MA, et al. Aberrant phenotypes in acute myeloid leukemia: a high frequency and its clinical significance. Haematologica. 2001;86(8):801-6.

16. Launder TM, Bray RA, Stempora L, Chenggis ML, Farhi DC. Lymphoidassociated antigen expression by acute myeloid leukemia. American journal of clinical pathology. 1996;106(2):185-91.

17. Chirumbolo S, Ortolani R, Vella A. CCR3 as a single selection marker compared to CD123/HLADR to isolate basophils in flow cytometry: some comments. Cytometry Part A : the journal of the International Society for Analytical Cytology. 2011;79(2):102-6.

18. Seegmiller AC, Kroft SH, Karandikar NJ, McKenna RW. Characterization of immunophenotypic aberrancies in 200 cases of B acute lymphoblastic leukemia. American journal of clinical pathology. 2009;132(6):940-9.

19. Alkayed K, Khattab E, Madanat F. Aberrant T-cell antigen expression in Jordanian children with B lymphoblastic leukemia. Hematology/oncology and stem cell therapy. 2015;8(4):187-8.

20. Venditti A, Del Poeta G, Buccisano F, Tamburini A, Cox-Froncillo MC, Aronica G, et al. Prognostic relevance of the expression of Tdt and CD7 in 335 cases of acute myeloid leukemia. Leukemia. 1998;12(7):1056-63.

21.Bradstock K, Matthews J, Benson E, Page F, Bishop J. Prognostic value of immunophenotyping in acute myeloid leukemia. Australian Leukaemia Study Group. Blood. 1994;84(4):1220-5.

22. Khurram M, Jafri S, Mannan A. Frequency Of Aberrant Expression Of Cd Markers In Cases Of Acute Leukemia. Medical Journal of Islamic World Academy of Sciences 2010; 18(2):55-60. 